\title{
Comparison of effectiveness and survival after the MitraClip or Carillon procedure for severe functional mitral regurgitation: a single-center retrospective analysis
}

\author{
Stephan Heyl, Aria Nikkhoo, Markus Wieszner, Stephan Fichtlscherer, Florian Seeger, \\ Birgit Assmus, Brigitte Luu, Katrin Hemmann, Claudia Walther, Joerg Honold
}

Department of Cardiology, Franfurt University Hospital, Frankfurt, Germany

Submitted: 11 May 2020

Accepted: 7 June 2020

Arch Med Sci Atheroscler Dis 2020; 5: e171-e177 DOI: https://doi.org/10.5114/amsad.2020.97160

Copyright (c) 2020 Termedia \& Banach

\section{Abstract}

Introduction: Current studies suggest improved survival in patients with severe functional mitral regurgitation (FMR) treated successfully with the MitraClip (MC) compared to medical treatment alone, in addition to a significant reduction of FMR severity. Recently, the Carillon system (CS) has also been shown to significantly reduce FMR. However, whether this beneficial effect of CS also translates into a survival benefit comparable to the MC system has not been investigated so far. The aim of the study was to compare the course of FMR grade and mortality after MC or CS in a retrospective, non-randomized, single-center analysis.

Material and methods: A hundred and fifty-four patients with symptomatic FMR $2+$ were included in this study (MC: $n=117, C S: n=37$ ). Baseline characteristics did not differ significantly between groups.

Results and conclusions: Initially, the degree of FMR was reduced in the MC group from $2.9 \pm 0.3$ to $1.7 \pm 0.7$ and from $2.7 \pm 0.5$ to $2.1 \pm 0.7$ in the CS group, $p$ within and between groups $<0.01$. Within 6 months, FMR remained reduced in the MC group $(1.83 \pm 0.6)$ and CS group ( $2.1 \pm 0.7)$. One-year survival was $34.8 \%$ in the MC group and $54.8 \%$ in the CS group $(p=0.663)$. Median long-term survival was 1.66 years in the MC group and 3.92 years in the CS group, log rank $p=0.001$.

Key words: survival, MitraClip, functional mitral regurgitation, Carillon.

\section{Introduction}

Functional mitral regurgitation (FMR) is a common finding in patients with heart failure due to reduced ejection fraction (HFrEF) [1]. One recent study reported a $20 \%$ prevalence of severe MR in HFrEF patients under optimal medical therapy (OMT) [2]. In HFrEF, severe FMR is associated with adverse outcome in terms of more severe heart failure symptoms, more frequent rehospitalization for heart failure and increased mortality [3].

In heart failure with preserved ejection fraction (HFpEF), FMR also occurs and is associated with adverse outcomes as well, independent from coexisting atrial fibrillation [4-6].

Current guidelines approve percutaneous edge-to-edge repair in patients with symptomatic FMR despite OMT after evaluation by the heart
Corresponding author:

Dr. Joerg Honold

Department of Cardiology

Franfurt University Hospital

Frankfurt, Country

E-mail: jhonold@gmx.net 
team with a level IIb C indication [1], based on early studies $[7,8]$. More recent data provide conflicting evidence whether the MitraClip (MC) procedure can attenuate heart failure and improve prognosis $[9,10]$. However, in a selected population of patients in the COAPT trial [9], MC was associated with significantly improved survival (33\% relative risk reduction for all-cause mortality), compared to FMR patients under OMT alone.

Catheter-based mitral annuloplasty with the Carillon system (CS) represents another option for percutaneous therapy in FMR [11]. So far, limited evidence regarding safety, feasibility and especially therapeutic effects of the CS device has been available [12, 13]. Recently, the randomized, blinded and sham-controlled Reduce-FMR trial from 2019 [14] assigned 120 patients with HFrEF and severe FMR to either OMT and a sham procedure or implantation of a CS device with blinded follow-up for mitral regurgitation volume and left ventricular dimensions. After 12 months, MR regurgitation volume decreased significantly, as well as left ventricular end-diastolic diameters. Moreover, a nonsignificant trend towards improved heart failure symptoms, reduced rehospitalization rates and mortality could be observed, although the study was neither designed nor powered for these endpoints.

Whether these competing devices, MC and CS, provide comparable effectiveness in terms of reduction of FMR and impact on prognosis has not been investigated separately so far in a head-tohead comparison study. Therefore, the aim of the present study was to describe the effectiveness in $M R$ reduction as well as all-cause mortality after either MC or CS as endpoints in a non-randomized single-center retrospective study.

\section{Material and methods}

\section{Patients}

Inclusion criteria for this single-center retrospective study were a history of interventional therapy for severe FMR or combined etiology with predominant functional MR by either MC or CS between 2013 and 2018 at Goethe University Hospital, Frankfurt, Germany. All patients suffered from symptomatic mitral regurgitation 2+ or 3 despite optimized medical therapy according to current guidelines at the time of invasive therapy. Assignment to the individual therapy was non-randomized and based on the choice of the operating cardiologist after an interdisciplinary heart team consensus favoring an interventional approach.

Exclusion criteria for CS were a history of or indication for cardiac resynchronization therapy with a left ventricular pacing lead in the coronary sinus, calcified mitral annulus, or prior mitral valve surgery. Exclusion criteria for MC were defined according to the consensus paper on AV valve therapy of the German Cardiac Society [15].

All patients gave their written informed consent on data collection and publication. The study was approved by the local ethics committee (protocol No. 512/15). The study was conducted according to the Declaration of Helsinki of 1975.

Patients were followed by telephone calls, contact with their general practitioner or request at a civil registry office.

\section{Methods}

Echocardiographic studies at baseline and follow-up were obtained according to the guidelines of the European Society of Cardiology [16], quantifying MR in three grades of severity by integrative assessment of qualitative, semi-quantitative and quantitative parameters.

In brief, MC was performed under general anesthesia after transfemoral venous access and transseptal puncture with access to the left atrium by TOE-guided grasping of the mitral leaflets with the MitraClip device and creating a novel double mitral orifice in order to reduce the severity of FMR [7].

CS was performed in conscious sedation by transjugular retrograde venous access of the coronary sinus and placement of an individually sized device for approximation of the mitral leaflets by reducing the mitral anulus diameter. Simultaneously, coronary angiography of the left circumflex and right coronary artery was performed in order to detect any significant coronary impingement by the device [11].

Echocardiographic follow-up was performed immediately after the procedure, and 6 months thereafter. Information about the vital status 12 months after therapy and thereafter was obtained by phone calls with the patients or requests of survival to the local authorities.

\section{Statistical analysis}

All statistical analysis was performed with SPSS 13.0.

If not depicted otherwise, continuous variables are given as mean \pm standard error, categorical variables as frequencies and percentages. Differences between variables were calculated using the $\chi^{2}$ test for categorical variables and one-way ANOVA test for continuous variables. Multivariate analysis was performed by stepwise forwards Cox proportional hazards ratio regression analysis. All $p$-values are reported two-sided with a level of significance of $p<0.05$. The Kaplan-Meier estimator was used for survival analysis and the log rank test. 


\section{Results}

$N=117$ patients underwent treatment with the MitraClip, $n=37$ patients were treated with the Carillon. No patients were lost to follow-up.

\section{Patient characteristics and procedural data}

Baseline characteristics of the two groups are depicted in Table I.
Patients in the CS group were significantly more often male, with a lower number of implanted ICDs. Ischemic heart disease was the leading cause of functional MR in both groups. Guideline-directed heart failure medication was uptitrated to the maximum tolerable dose in both groups before device implantation.

Both severity of FMR and degree of heart failure were more pronounced in the MC group, regarding lower left ventricle ejection fraction (LVEF)

Table I. Baseline characteristics

\begin{tabular}{|c|c|c|c|}
\hline Parameter & Carillon $(n=37)$ & MitraClip $(n=117)$ & $P$-value \\
\hline Female gender & $20(54.1)$ & $34(29.1)$ & 0.005 \\
\hline Age [years] & $72.4 \pm 12.3$ & $75.0 \pm 11.1$ & 0.36 \\
\hline $\mathrm{BMI}\left[\mathrm{kg} / \mathrm{m}^{2}\right]$ & $27.4 \pm 5.3$ & $27.0 \pm 5.1$ & 0.91 \\
\hline EURO-Score II & $13.4 \pm 13.4$ & $9.8 \pm 8.2$ & 0.33 \\
\hline LVEF (\%) & $41 \pm 17$ & $37 \pm 16$ & 0.20 \\
\hline Coronary artery disease & $23(62.2)$ & $60(51.2)$ & 0.53 \\
\hline History of CABG & $9(24.3)$ & $23(19.7)$ & 0.54 \\
\hline ICD & $8(21.6)$ & $48(41.0)$ & 0.032 \\
\hline \multicolumn{4}{|l|}{ Vital parameters: } \\
\hline Systolic BP [mm Hg] & $122 \pm 25$ & $122 \pm 20$ & 0.47 \\
\hline Diastolic BP [mm Hg] & $71 \pm 13$ & $69 \pm 11$ & 0.35 \\
\hline Heart rate [beats/min] & $67 \pm 15$ & $72 \pm 12$ & 0.21 \\
\hline \multicolumn{4}{|l|}{ Laboratory parameters: } \\
\hline NT-proBNP [pg/ml] & $2985 \pm 2896$ & $4374 \pm 8094$ & 0.46 \\
\hline Hemoglobin [mg/dl] & $12.2 \pm 2.1$ & $12.1 \pm 2.1$ & 0.48 \\
\hline GFR $[\mathrm{ml} / \mathrm{min}]$ & $51.8 \pm 20.3$ & $52.2 \pm 21.8$ & 0.45 \\
\hline \multicolumn{4}{|l|}{ Medication: } \\
\hline ASA & $12(38.7)$ & $38(33.9)$ & 0.62 \\
\hline ACEI or ARB & $28(90.3)$ & $96(85.7)$ & 0.77 \\
\hline$\beta$-blocker & $31(87)$ & $93(83.0)$ & 0.21 \\
\hline MRA diuretic agent & $11(35.5)$ & $53(47.3)$ & 0.24 \\
\hline Diuretic agents & $37(100)$ & $103(88)$ & 0.047 \\
\hline \multicolumn{4}{|l|}{ Echocardiographic parameters: } \\
\hline Mitral regurgitant grade & $2.7 \pm 0.5$ & $2.9 \pm 0.3$ & 0.006 \\
\hline Vena contracta width [mm] & $5.8 \pm 1.3$ & $6.5 \pm 1.3$ & 0.015 \\
\hline EROA $\left[\mathrm{cm}^{2}\right]$ & $0.36 \pm 0.17$ & $0.43 \pm 0.15$ & 0.026 \\
\hline Mitral regurgitant volume [ml/beat] & $56.3 \pm 34.1$ & $63.5 \pm 23.2$ & 0.07 \\
\hline LVEDD [mm] & $56.1 \pm 8.1$ & $62.1 \pm 10.4$ & 0.01 \\
\hline Tricuspid regurgitant grade & $2.0 \pm 0.7$ & $1.8 \pm 0.8$ & 0.22 \\
\hline Systolic PAP [mm Hg] & $50.6 \pm 9.8$ & $51.9 \pm 15.7$ & 0.77 \\
\hline
\end{tabular}

Data are presented as $n(\%)$ or mean \pm standard deviation. ACE - angiotensin-converting enzyme inhibitor, ARB - angiotensin-receptor blocker, $B M I$ - body mass index, $B P$ - blood pressure, $C A B G$ - coronary artery bypass graft surgery, EROA - effective regurgitant orifice area, GFR - glomerular filtration rate estimated by MDRD formula, ICD - implantable cardioverter-defibrillator, $L V E F$ - left ventricular ejection fraction, LVEDD - left ventricular end-diastolic diameter, MRA - mineralocorticoid receptor antagonist, NT-proBNP-N-terminal pro-B-type natriuretic peptide, NYHA - New York Heart Association, PAP - pulmonary artery pressure. 


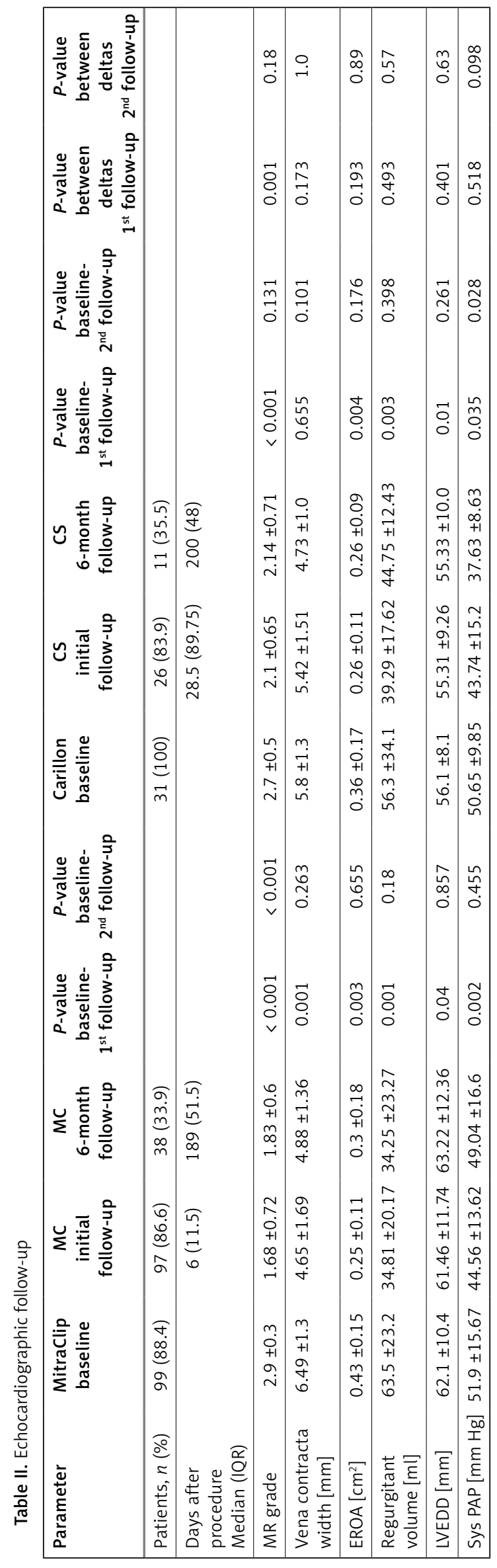

and higher NT-proBNP values as well as slightly but significantly higher grades of MR reflected by vena contracta width, effective regurgitant orifice area (EROA), regurgitant volume and left ventricular end-diastolic diameter (LVEDD) (Table I) in the MC group.

Device implantation succeeded in 112 (95.7\%) patients of the MC group and 31 (83.8\%) patients of the CS group $(p=0.024)$. The lower rate of procedural success in the CS group was due to impingement of a relevant branch of the left circumflex artery, dissection of the coronary sinus or unavailability of a suitable device for individual CS anatomy.

\section{Echocardiographic follow-up}

First echocardiographic follow-up after therapy was obtained in 97 (86.8\%) patients of the MC group after a median of 6 days (IQR $=11.5$ days) and 26 (83.9\%) patients of the CS group (Table II) after a median of 28.5 days (IQR $=89.8$ days) (Table II).

In both groups, MR degree, EROA and regurgitation volume as well as systolic pulmonary artery pressure were significantly reduced. Moreover, LVEDD diminished significantly in both groups as a marker of inverse remodeling. Although the grade of FMR was statistically significant between the MC and CS group in this short-term follow-up, no significant differences between the deltas of the single echocardiographic parameters vena contracta width, EROA and systolic pulmonary artery pressure between the MC and CS group were detected (Table II).

Echocardiographic follow-up at 6 months was available in 38 patients of the MC group (33.9\%) and 11 patients of the CS group (35.5\%) after a median of $189($ IQR $=51.5)$ days and 200 (IQR 48) days, respectively (Table II).

In both groups, grade of MR as well as vena contracta width, EROA and regurgitation volume remained reduced compared to baseline parameters, with an increase in LVEDD in the MC group. Systolic PA pressure showed a significantly more pronounced reduction in the CS group, compared to the MC group (Table II).

For the whole study population as well as the subgroup of patients treated with MC, no predictors of procedural success defined as reduction of FMR by at least one grade could be detected. Only in the subgroup analysis of the CS group was a higher grade of pre-interventional tricuspid regurgitation significantly associated with therapeutic failure despite proper device implantation (patients with MR reduction by one grade or more: TR $1.1 \pm 0.7$ versus a TR of $1.9 \pm 0.6$ in patients with MR reduction of less than one grade, $p=0.005)$. 


\section{Survival during follow-up}

Within 12 months after therapy, 14 patients of the CS group and 73 patients of the MC group died, accounting for a 1-year mortality of $45.2 \%$ and $65.2 \%$, respectively (Figure 1 ).

During follow-up of up to 5 years, survival curves diverged significantly, with a median survival of 1.66 years in the MC group and 3.92 years in the Carillon group, $p=0.01$ in log rank testing. Five-year survival rates were $0 \%$ in the MC group and $6.5 \%$ in the Carillon group.

Univariate analysis of baseline demographic parameters and survival revealed that end stage renal disease necessitating hemodialysis was significantly associated with adverse outcome in the MC group. Only 2 of the 10 MC patients with hemodialysis at baseline reached median survival, whereas 8 patients died before $(p=0.057)$.

In multivariate analysis including all parameters significantly different between the Carillon and MitraClip groups at baseline, only left ventricular end-diastolic diameter persisted as an independent predictor of mortality during follow-up (hazard ratio $=1.06,95 \%$ confidence interval: 1.015-1.056, $p=0.012$ ).

\section{Discussion}

This is the first study to describe the differential effects of MC or CS on the degree of functional mitral regurgitation and long-term survival in an unselected, non-randomized all-comers population. However, due to the limitations described below, its contribution is predominantly hypothesis-generating.

Our study cohort displays similar characteristics comparable to populations of FMR patients examined in large contemporary randomized trials $[9,10]$. Moreover, the reduction of MR severity corresponds to the therapeutic effects of both devices described before [9, 14].

Due to the limited number of follow-up examinations, we cannot provide a complete course of FMR grade especially for the CS group, which might be of particular interest as the CS system is well described to bear later responsiveness in FMR reduction, potentially dependent on the angle between the coronary sinus and mitral valve plane [17].

The 1-year mortality rates of $45.2 \%$ and $65.2 \%$ in the CS and MC groups of our study are higher than all-cause mortality seen in COAPT with $19.1 \%$ in the device group and $23.2 \%$ in the control group. A recent analysis of pooled Carillon data reports $83.6 \% 1$-year survival and $56.2 \%$ survival after 4 and 5 years [18].

This difference in mortality can only partially be explained by the introduction of angioten- sin receptor neprilysin inhibitor (ARNI) for pharmacological therapy in contemporary heart failure patients [19-21], its effects on FMR [22] or emerging usage of defibrillator/resynchronization therapy [23]. However, a mean EuroSCORE II [24] of 10.7 in patients with pronounced left ventricular remodeling reflected by a mean LVEF of $37 \%$ and a mean LVEDD of $61 \mathrm{~mm}$ with FMR grade 3 displays a cardiac high-risk collective at the time of treatment between 2013 and 2018 that might not have been included in a contemporary randomized trial.

The striking difference in all-cause mortality between the MC and CS groups during the longterm follow-up has to be interpreted with caution. As pointed out before, only limited numbers of follow-up echocardiographic examinations are available (Table II). In a subset of CS patients $(n=9)$ with long-term follow-up up to 2 years, FMR remained reduced to an average $\mathrm{MI}$ grade of $1.94 \pm 0.63$. We cannot provide similar follow-up data for the MC group. As the vast majority of MC and CS patients did not undergo prespecified echocardiographic long-term follow-up, we cannot exclude that a substantial proportion of patients from any therapy group deteriorated in FMR grade over time with a potential adverse impact on survival. Due to the limited follow-up in our cohort, we cannot reproduce the beneficial impact of successful CS therapy within short-term follow-up on long-term survival as recently described [18]. However, in multivariate analysis, left ventricular end diastolic diameter persisted as an independent predictor of mortality, indicating that the differences in mortality are mainly driven by selection bias of patients, with more pronounced heart failure in the MC group.

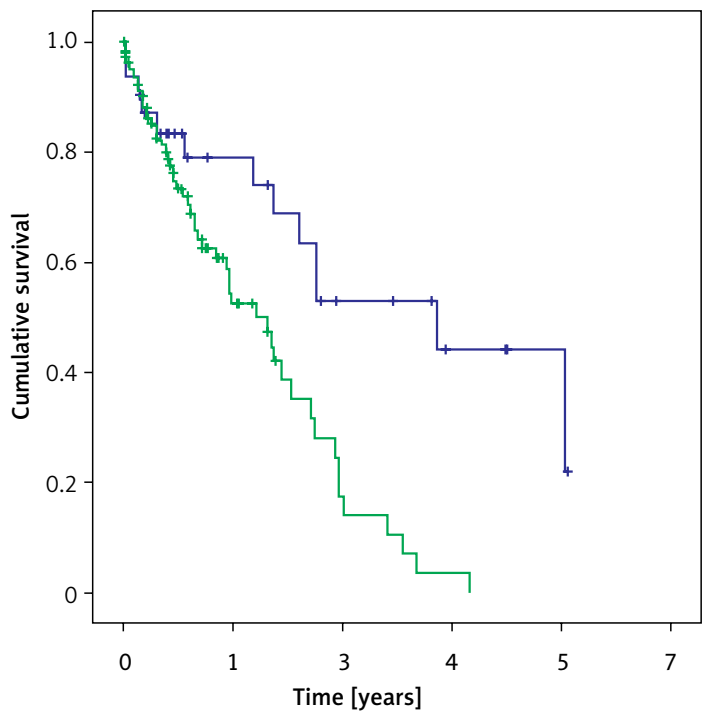

Figure 1. Kaplan-Meier plot of cumulative survival in years for the MitraClip group (green line) and Carillon group (blue line). Log rank $p$ was 0.001 
This study bears a number of substantial limitations to be taken into account when interpreting the data:

Due to the retrospective design of the study with uncoordinated echocardiographic follow-up, we were not able to provide full information about the course of mitral regurgitation after therapy in all patients for both groups. Allocation of patients to either MC or CS was not randomized but based on operators' choice, potentially influenced by personal experience and facilities' capacity for either MC or CS at the time of therapy. Both therapy groups included patients first to be treated with $M C$ and CS at the institution. We can therefore not exclude that contemporary patients might benefit more from therapy as the learning curve for either therapy has to be taken into account for procedural success.

The impact of both therapies on the degree and course of heart failure is an important issue. Due to the lack of data, we are not able to provide important heart failure parameters such as NYHA class, NT-proBNP levels or 6-minute walking tests during follow-up.

We can only provide overall mortality and have no insights into the causes of death, especially cardiovascular mortality. The sample size of the study population does not allow robust conclusions about the differential impact on mortality by the examined treatments for MR.

In conclusion, the less invasive Carillon approach appears to bear comparable beneficial short-term effects on the grade of FMR and LV remodeling and a comparable 1-year survival outcome, compared to the MitraClip.

As interventional mitral valve therapies are emerging, differential effects and long-term clinical and survival efficacies of evidence-based device therapies such as the MitraClip and Carillon have to be investigated in properly randomized upcoming clinical trials.

\section{Conflict of interest}

The authors declare no conflict of interest.

\section{References}

1. Falk V, Baumgartner H, Bax J, et al. 2017 ESC/EACTS VHD Guidelines. Eur J Cardiothorac Surg 2017; 52: 616-64.

2. Goliasch G, Bartko PE, Pavo N, et al. Refining the prognostic impact of functional mitral regurgitation in chronic heart failure. Eur Heart J 2018; 39: 39-46.

3. De la Espriella R, Santas E, Miñana G, et al. Functional mitral regurgitation predicts short-term adverse events in patients with acute heart failure and reduced left ventricular ejection fraction. Am J Cardiol 2017; 120: 1344-8.

4. Tamargo M, Obokata M, Reddy YNV, et al. Functional mitral regurgitation and left atrial myopathy in heart failure with preserved ejection fraction. Eur J Heart Fail 2020; 22: 489-98.

5. Abe Y, Takahashi Y, Shibata T. Functional mitral regurgitation, updated: ventricular or atrial? J Echocardiogr 2020; 18: 1-8.

6. Deferm S, Bertrand PB, Verbrugge FH, et al. Atrial functional mitral regurgitation: JACC review topic of the week. J Am Coll Cardiol 2019; 73: 2465-76.

7. Feldman T, Kar S, Rinaldi M, et al. Percutaneous mitral repair with the mitraclip system. safety and midterm durability in the initial EVEREST (Endovascular Valve Edge-to-Edge REpair Study) cohort. J Am Coll Cardiol 2009; 54: 686-94.

8. Glower DD, Kar S, Trento A, et al. Percutaneous mitral valve repair for mitral regurgitation in high-risk patients: results of the EVEREST II study. J Am Coll Cardiol 2014; 64: 172-81.

9. Stone GW, Lindenfeld J, Abraham WT, et al.; COAPT Mitraclip. Transcatheter mitral-valve repair in patients with heart failure. N Eng J Med 2018; 379: 2307-18.

10. Obadia JF, Messika-Zeitoun D, Leurent G, et al.; MITRA FR Mitraclip. Percutaneous repair or medical treatment for secondary mitral regurgitation. N Engl J Med 2018; 379: 2297-306.

11. Siminiak T, Firek L, Jerzykowska O, et al. Percutaneous valve repair for mitral regurgitation using the Carillon(trademark) Mitral Contour System. Description of the method and case report. Kardiol Pol 2007; 65: 272-8.

12. Lipiecki J, Siminiak T, Sievert $\mathrm{H}$, et al. Coronary sinus-based percutaneous annuloplasty as treatment for functional mitral regurgitation: the TITAN II trial. Open Heart 2016; 3: e0000411.

13. Nickenig G, Hammerstingl C, Schueler R, et al. Transcatheter mitral annuloplasty in chronic functional mitral regurgitation: 6-month results with the cardioband percutaneous mitral repair system. JACC Cardiovasc Interv 2016; 9: 2039-47.

14. Witte KK, Lipiecki J, Siminiak T, et al. a randomized sham-controlled study of percutaneous mitral annuloplasty in functional mitral regurgitation. JACC Hear Fail 2019; 7: DOI: 10.1016/j.jchf.2019.06.011.

15. Baldus S, Kuck KH, Rudolph V, et al. Interventional therapy for AV valve disease - focus on mitral valve regurgitation: position paper of the German Cardiac Society. Kardiologe 2018; 12: 128-44.

16. Lancellotti P, Tribouilloy C, Hagendorff A, et al. Recommendations for the echocardiographic assessment of native valvular regurgitation: an executive summary from the European Association of Cardiovascular Imaging. Eur Heart J Cardiovasc Imaging 2013; 14: 611-44.

17. Rottländer D, Schneider T, Degen H, Laufenberg M, Chatrou $M$, Haude $M$. Coronary sinus to mitral valve annulus topography and response to the percutaneous coronary sinus-based mitral valve contour system. Eurolntervention 2019; 15: 923-6.

18. Lipiecki J, Kaye DM, Witte KK, et al. Long-term survival following transcatheter mitral valve repair: pooled analysis of prospective trials with the Carillon device. Cardiovasc Revasc Med 2020; doi: 10.1016/j.carrev. 2020.02.012.

19. MCMurray JJV, Packer M, Desai AS, et al. Angiotensinneprilysin inhibition versus enalapril in heart failure. N Engl J Med 2014; 371: 993-1004.

20. Lamas GA, Mitchell GF, Flaker GC, et al. Clinical significance of mitral regurgitation after acute myocardial infarction. Circulation 1997; 96: 827-33.

21. Yancy CW, Jessup M, Bozkurt B, et al. 2017 ACC/AHA/ HFSA Focused Update of the 2013 ACCF/AHA Guideline 
for the Management of Heart Failure: A Report of the American College of Cardiology/American Heart Association Task Force on Clinical Practice Guidelines and the Heart Failure Society of Amer. Circulation 2017; 136: e137-61.

22. Kang DH, Park SJ, Shin SH, et al. Angiotensin receptor neprilysin inhibitor for functional mitral regurgitation. Circulation 2019; 139: 1354-65.

23. Priori SG, Blomstrom-Lundqvist C, Mazzanti A, et al. 2015 ESC Guidelines for the management of patients with ventricular arrhythmias and the prevention of sudden cardiac death: The Task Force for the Management of Patients With Ventricular Arrhythmias and the Prevention of Sudden Cardiac Death of the European Society of Cardiology (ESC). Endorsed By: Association for European Paediatric and Congenital Cardiology (AEPC). Eur Heart J 2015; 36: 2793-867.

24. Guida P, Mastro F, Scrascia G, Whitlock R, Paparella D. Performance of the European System for Cardiac Operative Risk Evaluation II: a meta-analysis of 22 studies involving 145,592 cardiac surgery procedures. J Thorac Cardiovasc Surg 2014; 148: 3049-57. 